\title{
Genomic analysis reveals Lactobacillus sanfranciscensis as stable element in traditional sourdoughs
}

\author{
Rudi F Vogel', Melanie Pavlovic ${ }^{1}$, Matthias A Ehrmann ${ }^{1 *}$, Arnim Wiezer ${ }^{3}$, Heiko Liesegang ${ }^{3}$, Stefanie Offschanka ${ }^{3}$, \\ Sonja Voget ${ }^{1}$, Angel Angelov ${ }^{2}$, Georg Böcker ${ }^{4}$, Wolfgang Liebl ${ }^{2}$
}

From 10th Symposium on Lactic Acid Bacterium

Egmond aan Zee, the Netherlands. 28 August - 1 September 2011

\begin{abstract}
Sourdough has played a significant role in human nutrition and culture for thousands of years and is still of eminent importance for human diet and the bakery industry. Lactobacillus sanfranciscensis is the predominant key bacterium in traditionally fermented sourdoughs.

The genome of L. sanfranciscensis TMW 1.1304 isolated from an industrial sourdough fermentation was sequenced with a combined Sanger/454-pyrosequencing approach followed by gap closing by walking on fosmids. The sequencing data revealed a circular chromosomal sequence of 1,298,316 bp and two additional plasmids, pLS1 and pLS2, with sizes of $58,739 \mathrm{bp}$ and $18,715 \mathrm{bp}$, which are predicted to encode 1,437, 63 and 19 orfs, respectively. The overall GC content of the chromosome is $34.71 \%$. Several specific features appear to contribute to the ability of $L$. sanfranciscensis to outcompete other bacteria in the fermentation. L. sanfranciscensis contains the smallest genome within the lactobacilli and the highest density of ribosomal RNA operons per Mbp genome among all known genomes of free-living bacteria, which is important for the rapid growth characteristics of the organism. A high frequency of gene inactivation and elimination indicates a process of reductive evolution. The biosynthetic capacity for amino acids scarcely availably in cereals and exopolysaccharides reveal the molecular basis for an autochtonous sourdough organism with potential for further exploitation in functional foods. The presence of two CRISPR/cas loci versus a high number of transposable elements suggests recalcitrance to gene intrusion and high intrinsic genome plasticity.
\end{abstract}

\section{Background}

The use of sourdough is documented for $>5,000$ years and of eminent industrial importance in the production of baked goods amounting to more than 3 million tons of baked goods annually [1]. Annual per capita consumption of baked goods in Europe is $50-85 \mathrm{~kg}$ with up to $20 \%$ involving sourdough fermentations with wheat or rye, i. e. a total of $>3$ million tons. To date, no bacterial genomes from strains adapted to this huge man made habitat in millions of generations are available. Lactobacillus sanfranciscensis was first described in 1971 by Kline and Sugihara who isolated and characterized obligately

\footnotetext{
* Correspondence: m.ehrmann@wzw.tum.de

'Lehrstuhl für Technische Mikrobiologie, Germany

Full list of author information is available at the end of the article
}

heterofermentative lactobacilli from San Francisco sourdoughs [2]. The name Lactobacillus sanfrancisco refers to the city where the sourdoughs from which the organism was isolated had been propagated for more than 100 years. At that time the species was not included in the Approved Lists of Bacterial Names and had no standing in bacteriological nomenclature until the name was revived by Weiss and Schillinger in 1984 [3]. To follow the Rules of the International Code of Nomenclature of Prokaryotes the species epithet was changed to L. sanfranciscensis [4]. Sourdough fermentations worldwide are characterized by a highly stable association of yeasts and lactic acid bacteria. In rye and wheat sourdoughs with a tradition of continuous propagation by back-slopping procedures, L. sanfranciscensis is the probably the most adapted species and regarded as

\section{() Biomed Central}

(c) 2011 Vogel et al; licensee BioMed Central Ltd. This is an open access article distributed under the terms of the Creative Commons Attribution License (http://creativecommons.org/licenses/by/2.0), which permits unrestricted use, distribution, and reproduction in any medium, provided the original work is properly cited. 
autochthonous key organism of the sourdough microbiota [5,6]. Its phylogenetic position within the genus lactobacillus is shown in Figure 1. Multiple metabolic activities of $L$. sanfranciscensis have been described in the literature that contribute to the quality of sourdough and baked goods. With the exception of one report by Groeneveld et al. [7], who allotted isolates from fruit flies exhibiting $97 \%$ rDNA sequence homologies as L. sanfranciscensis, this species has only been isolated from sourdoughs, while strains of all other species found in sourdough are frequently isolated also from other habitats. None of the genome-sequenced strains of these genera, e. g. L. plantarum or L. reuteri were isolated from sourdough. This raises the question for the role of man in evolution of $L$. sanfranciscensis.

The sourdough microbiota including $L$. sanfranciscensis contribute to dough rheology and flavour properties due to a strong acidification by an optimized carbohydrate metabolism and the liberation of precursors of volatile compounds by the proteolytic system [8-10] and the catabolism of specific amino acids $[5,10,11]$. Formation of exopolysaccharides (homopolysaccharides and fructooligosaccharides) enhance texture, shelf life and nutritional value [12]. The sequenced strain $L$. sanfranciscensis TMW 1.1304 was isolated in 2006 from a commercial mother sponge with a tradition of continuous propagation. The presence of this strain in this sourdough starter was demonstrated over a period of at least 20 years, and it accounts for more than $90 \%$ of the microflora of that product.

\section{Results and discussion}

General genomic features

The L. sanfranciscensis TMW 1.1304 genome project allowed the assembly of a circular chromosomal sequence

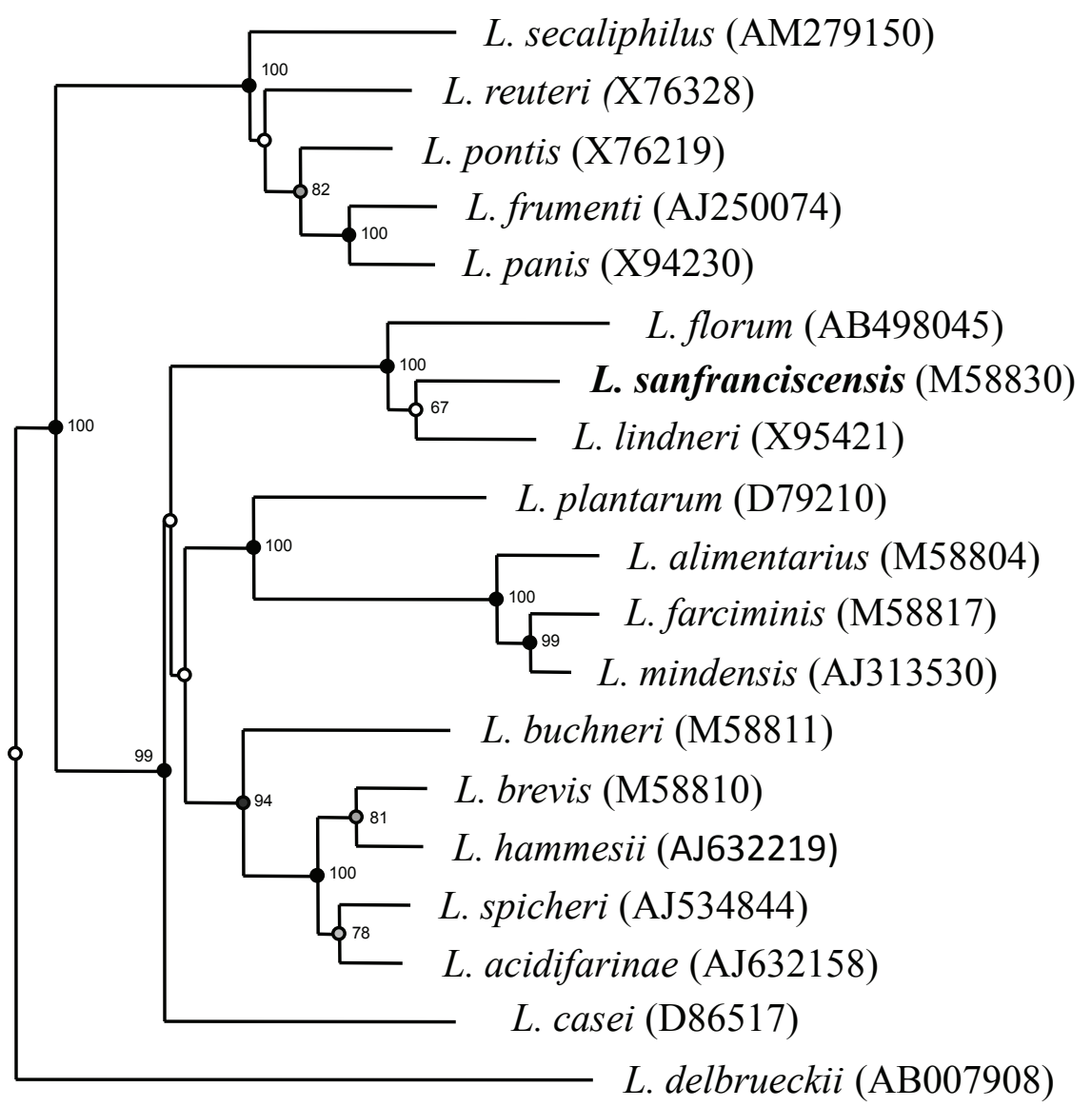

0.01

Figure 1 Neighbour joining phylogenetic tree of Lactobacillus species showing the phylogenetic position of Lactobacillus sanfranciscensis based on 165 rRNA gene sequences. The scale bar indicates 1 nucleotide substitution per 100 nucleotides. Numbers in parentheses indicate accession numbers of 165 rRNA genes from type strains. Bootstrap values over 50\% (based on 100 replications) are shown at the noodes. 
of 1,298,316 bp and two additional plasmids of 58,739 bp and $18,715 \mathrm{bp}$. The genome size of strain TMW 1.1304 was estimated at approximately $1.3 \mathrm{Mbp}$ on the basis of results obtained by pulsed-field gel electrophoresis of chromosomal DNA restriction fragments. A previous estimation of the apparent genome size based on four strains of $L$. sanfranciscensis isolated from Italian sourdoughs and the type strain DSM $20451^{\mathrm{T}}$ indicates 1.4 Mbp [13]. Thus, the $L$. sanfranciscensis genome is the smallest genome within the genus Lactobacillus so far followed by the recently published genome of Lactobacillus iners AB-1 with 1,304 Mbp [14]. The general features of the sequence are presented in Table 1. The average orf length is 835 and the codon density is 87.1 , which is in the range of other lactobacilli (Additional file 1).

On the basis of analysis of the GC skew $(\mathrm{G}-\mathrm{C} / \mathrm{G}+\mathrm{C})$, the cumulative GC-skew and the location of characteristic genes (chromosomal replication initiator protein DnaA) we could identify a typical bacterial origin of replication and its beginning was assigned as base-pair one of the genome. Thus, two equal replication arms (replichores) were present and the locations of the predicted 1,437 coding sequences on the two strands correlated well with the direction of replication (Fig. 2). There were 153 pseudogenes found randomly distributed in the chromosome. Genes encoding replication functions overlap sequences with significant changes in GC skew indicating the location of the origin of replication (oriC). This region harbors the genes for the replication initiator protein $(d n a \mathrm{~A})$, the beta subunit of DNA polymerase III $(d n a \mathrm{~N})$, and the DNA gyrase subunits $\mathrm{A}$ and $\mathrm{B}$ ( $g y r \mathrm{~A}$ and $g y r \mathrm{~B})$. The arrangement of these genes, $d n a \mathrm{~A}-d n a \mathrm{~N}-r e c \mathrm{~F}-g y r \mathrm{~B}-g y r \mathrm{~A}$, is similar to that found in other Gram-positive bacterial genomes studied so far. Five DnaA-box consensus sequences
(TTATNCACA) were found upstream of $d n a \mathrm{~A}$ and three in the $d n a \mathrm{~A}-d n a \mathrm{~N}$ intergenic region. Opposite of the genome between position 628,700 and 628,800 a second change in GC skew indicates the replication terminus.

\section{Stable RNA gene density and codon usage bias}

Seven rRNA operons and 61 tRNA genes were detected, demonstrating an intriguingly high density of genes for stable RNAs in the genome of $L$. sanfranciscensis TMW 1.1304. Analysis of approx. 1000 complete genomes (Additional file 2) available in GenBank revealed that L. sanfranciscensis TMW 1.1304 has the highest rRNA operon density (5.39 per Mbp) among all known free-living organisms. The only genome with a higher rRNA gene density is that of Candidatus Carsonella ruddii PV (1 rRNA operon in its 0,159662 Mbp genome), an obligate insect endosymbiont not capable of autonomous growth whose status as a living organism is debatable [15] due to the lack of most replication, transcription and translation genes considered as essential for living cells. Interestingly, $50 \%$ of the top 20 species with the highest rRNA gene densities (rRNA gene densities above 2.9 per Mbp; species represented by more than one sequenced genome were only counted once; genomes from nonfree-living bacteria with canditatus status were disregarded) were lactic acid bacteria, i. e. various Lactobacillus and Streptococcus species (Additional file 3).

Multiple rRNA operons, which are found in many prokaryotes, may be of importance to achieve high growth rates and to adapt rapidly to changing environmental conditions $[16,17]$. We postulate that the exceptionally high rRNA operon density on the L. sanfranciscensis genome allows the bacteria to respond quickly to favorable growth conditions in their sourdough environment,

Table 1 General features of the $L$. sanfranciscensis genome compared with genomes of other species, which are found in sourdough (however, the strains whose genomes have been sequenced were isolated from other sources). Data are from this study and [59]

\begin{tabular}{|c|c|c|c|}
\hline & L. sanfranciscensis TMW 1.1304 & L. reuteri JCM $1112^{\top}$ & L. fermentum IFO 3956 \\
\hline Chromosome size (bp) & $1,298,316$ & $2,039,414$ & $2,098,685$ \\
\hline Plasmid(s) & 58,739 and 18,715 & - & - \\
\hline GC content (\%) & $34.7(37.6 \text { and } 36,1)^{*}$ & 38.9 & 51.5 \\
\hline Total ORFs & $1,437(63+19)$ & 1,820 & 1,844 \\
\hline Functionally assigned & 791 & 1,211 & 1,212 \\
\hline Conserved hypothetical & 498 & 413 & 360 \\
\hline Non-conserved hypothetical & 148 & 196 & 272 \\
\hline Coding density (\%) & 88.1 & 83.6 & 80.4 \\
\hline tRNAs & 61 & 58 & 54 \\
\hline rRNA operons & 7 & 6 & 5 \\
\hline Phage-related ORFs & not detected & 53 & 24 \\
\hline Transposases & 111 & 55 & 106 \\
\hline Group II introns & not detected & 12 & 0 \\
\hline
\end{tabular}

* values in brackets are for the plasmids pLS1 and pLS2, respectively. 


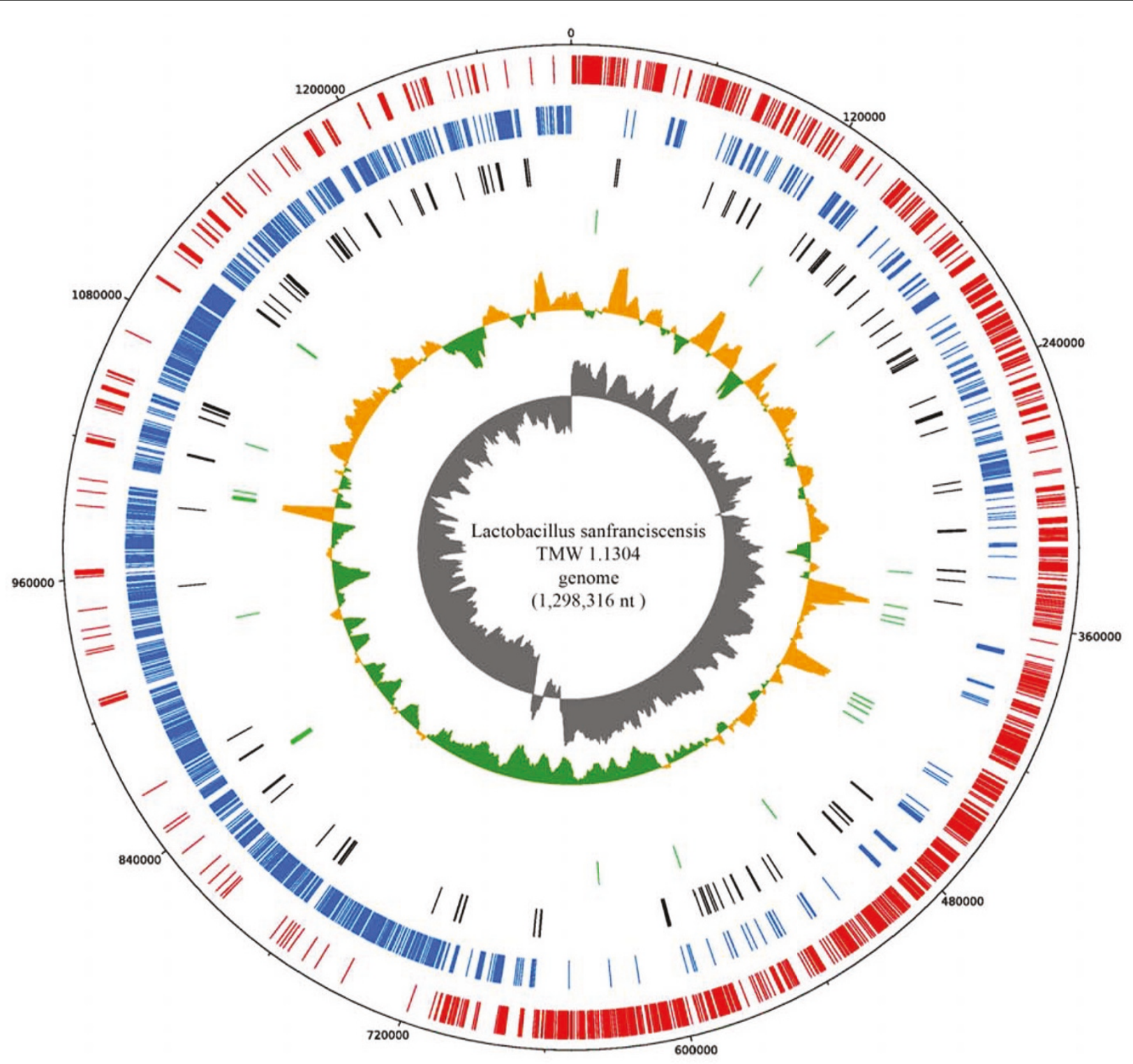

Figure 2 Genomic atlas of L. sanfranciscensis TMW 1.1304. From the outer circle inward, CDS on the forward strand (red), CDS on the reverse strand (blue), Pseudogenes on both strands (black), tRNA genes (green), GC content deviations from the average GC-content (green, low GC spike and orange, high GC spike), GC skew (grey). The GC\% and GC skew (C-G)/(C+G) were calculated in a window of $10000 \mathrm{nt}$, in steps of $200 \mathrm{nt}$.

rapidly initiating fermentative metabolism and fast growth which in combination with their specifically adapted metabolism (see below) could help to out-compete other contaminating bacteria. This strategy may also hold true for other lactic acid bacteria with a high rRNA operon density such as Lactobacillus delbrueckii subsp. bulgaricus (second-ranking after L. sanfranciscensis with an rRNA operon density of $4.85 / \mathrm{Mbp}$ of strain ATCC BAA-365) which is one of the classical starter organisms responsible for rapid lactic fermentation during yoghurt production.
Numerous microbial genomes reveal a codon usage bias (CUB), i.e. a pronounced preference for a specific set of codons (named major codons), in genes whose products are required in large quantities, which improves translation efficiency of these genes and contributes to optimizing cell growth $[18,19][20]$. The L. sanfranciscensis genome revealed a relatively strong CUB. A closer look at the set of genes that are translationally optimized in this organism revealed that the top 80 hits expectedly contained many genes (44) encoding ribosomal proteins and translation factors (Additional 
file 4), but also with the exception of the ribulose-5phosphate epimerase gene all genes for the formation of lactate, $\mathrm{CO}_{2}$ and ethanol via the phosphoketolase pathway, underscoring the importance of the efficient expression of this pathway for $L$. sanfranciscensis.

\section{Carbohydrate metabolism}

Consistent with the classification of $L$. sanfranciscensis as a heterofermentative lactic acid bacterium (LAB) all genes required for the phosphoketolase pathway are present in the $L$. sanfranciscensis genome whereas no homologues to transaldolase or transketolase were found. In silico analyses revealed that the sequenced $L$. sanfranciscensis strain is likely to use maltose, fructose, ribose and gluconate as carbon sources (Additional file 5). Additionally, two copies of a transporter for arabinose were found (LSA_1450, LSA_1460) of which only one seems to be functional as LSA_1460 is truncated at the 3' end. The presence of two genes for oligo-1,6-glucosidase (LSA_05810; LSA_01770) indicates the ability to hydrolyse $\alpha-1,6-\mathrm{D}$-glucosidic linkages in oligosaccharides produced from starch and glycogen (isomaltulose, isomaltotriose, panose and isomaltose). Except for maltose phosphorylase (LSA_01510) and a truncated alpha-glucosidase (LSA_05800) no additional ORFs for glycoside hydrolases were annotated.

Growth of L. sanfranciscensis with maltose as carbon source is generally accelerated when fructose, citrate or $\alpha$ ketoglutarate are used as alternative electron acceptors. $[21,22]$. Several uptake systems for possible electron acceptors were present. Fructose can be transported by a fructose permease (LSA_2810) and reduced to mannitol using a mannitol-2-dehydrogenase (LSA_02820). Two genes for citrate-sodium symporters (LSA_08630 and LSA_13030) and one gene encoding a malate uniport protein (LSA_02110) suggest uptake of citrate and malate. Genes necessary to reduce citrate to lactate are also present (LSA_12980 and LSA_12990 for citrate lyase, LSA_13020 for oxaloacetate decarboxylase, while no homologue gene for a succinate dehydrogenase which is necessary for the utilization of malate as electron acceptor was found.

The use of $\alpha-K G$ as electron acceptor by conversion to 2-hydroxyglutarate by LAB was previously mentioned by Radler and Broehl, 1984[23]. Recently, reduction of $\alpha$-ketoglutarate to 2-hydroxyglutarate was demonstrated in L. sanfranciscensis indicating that $\alpha$-ketoglutarate was used preferably as electron acceptor and NADH-dependent hydroxyglutarate dehydrogenase activity was confirmed by enzymatic analysis of crude cell extracts of L. sanfranciscensis[22]. Several orfs with putative $\alpha$-hydroxy acid dehydrogenase activity were present.

As expected, the genome of $L$. sanfranciscensis only encodes an incomplete citrate cycle as only genes for fumarate hydratase (LSA_12040), malate dehydrogenase
(LSA_02100; LSA_04670) and citrate lyase (LSA_12980 and LSA_12990) are present.

\section{Pyruvate metabolism}

Due to a frameshift in the pyruvate oxidase gene (EC 1.2.3.3/ LSA_00220) direct conversion of pyruvate to acetyl phosphate is not possible for L. sanfranciscensis. Therefore, the organism most likely converts pyruvate to acetate via lactate first and then generates acetyl phosphate from acetate. Enzymes required for redundant pathways like formate $\mathrm{C}$-acetyltransferase (EC 2.3.1.54) or acetaldehyde dehydrogenase (EC 1.2.1.10) were not encoded by the $L$. sanfranciscensis genome but in lactobacilli with larger genomes like L. plantarum or L. casei. In spite of the presence of only a relative low number of pyruvate dissipating enzymes in the $L$. sanfranciscensis genome, a high degree of redundancy for lactate dehydrogenase $(l d h)$ encoding genes was observed as at least three L-lactate dehydrogenases (LSA_09870, LSA_11450, LSA_13040) and three D-lactate dehydrogenases (LSA_00860, LSA_10990, LSA_12510) were found, among which LSA_11450 and LSA_12510 are pseudogenes. The presence of several copies of $l d h$ genes in other lactobacilli, e.g. L. plantarum [24] or L. casei ATCC 334 [25] in connection with the broad range of substrate selectivity described for those enzymes stresses their key function of $\mathrm{NAD}^{+}$ regeneration. Pyruvate can be produced by L. sanfranciscensis from a number of substrates. Besides the usual formation from sugars and gluconate via the phosphoketolase pathway, pyruvate can be generated from asparagine and alanine via transamination and from malate catalysed by malate dehydrogenase (LSA_02100, EC 1.1.1.38).

\section{Formation of exopolysaccharides (EPS)}

Formation of EPS is a trait often found in lactic acid bacteria [26]. Heterofermentative lactobacilli occurring in sourdough mostly synthesize glucan or fructan homopolymers. These are formed from sucrose by secreted or cell-anchored glucosyltransferases, which convert the sucrose into high-molecular-weight polymers, with the concomitant release of the respective hexose.

In TMW 1.1304 two genes encode respective glucosytransferases, both carrying a LPXTG sortase recognition motif. A plasmid encoded dextransucrase (LSA_2p00510) with a best protein match (85\%) to a dextransucrase of $L$. reuteri JCM 1112 is obviously not active due to the lack of ca. 500 aa residues at the $\mathrm{N}$-terminus and an atypical small molecular weight. A levansucrase (LSA_LSA_02160) was found to be identical toa levansucrase previously described by Tieking et al. from L. sanfranciscensis TMW 1.392 [27]. Interestingly, a 48 aa residue deletion 
corresponding to 4 direct repeats (PVNPSQPTTPAK) in the PXX motif of the $\mathrm{C}$-terminal cell wall anchor is observed.

The production of EPS by TMW 1.1304 can be demonstrated by growing the strain on mMRS containing $80 \mathrm{~g} \mathrm{l}^{-1}$ sucrose. To analyze the type of the polymer, the EPS was precipitated by adding two volumes of ethanol (99\%) and incubation at $4^{\circ} \mathrm{C}$ for $18 \mathrm{~h}$. EPS was hydrolyzed with perchloric acid at $100^{\circ} \mathrm{C}$ for $3 \mathrm{~h}$ and sugar monomers were analyzed with HPLC as described by Waldherr et al, 2008 [28]. Under these conditions the EPS produced by TMW 1.1304 was demonstrated to consist of fructose, indicating that EPS is a high molecular fructan and the levansucrase is functional.

Besides their role for bacterial metabolism, for which protective functions and an altered metabolite profile as a result of alternative use of electron acceptors are discussed, exopolysaccharides impact on crumb structure and shelf life of sourdough breads. Fructans and fructooligosaccharides likely produced by $L$. sanfranciscensis TMW 1.304 may serve further functional aspects in nutrition and medicine [29].

\section{Amino acid metabolism}

In silico analyses of the genome of $L$. sanfranciscensis TMW 1.1304 indicate the potential to synthesize de novo four amino acids (alanine from pyruvate, aspartate from oxaloacetate, glutamate and glutamine. L-alanine can be converted into L-cysteine using a cysteine desulfurase (EC 2.8.1.7, LSA_0990), while arginine, lysine and asparagine result from conversion pathways of L-aspartate. Therefore, L. sanfranciscensis is auxotroph for the remaining 12 amino acids (Table 2). As concentrations in wheat of aspartate, asparagine and glutamate are low, preservation of the biosynthetic pathways for these suggests adaptation to the sourdough environment.

\section{Purine and pyrimidine biosynthesis}

The enzyme required to generate 5-phosphoribosyl-1pyrophosphate (PRPP) from the phosphoketolase pathway intermediate ribulose-5-phosphate (EC 5.3.1.6/ LSA_04470 and EC 2.7.6.1/ LSA_04050; LSA_09930) are present in L. sanfranciscensis. As described for L. gasseri [30] six of the subsequent nine enzymes required to generate IMP from PRPP seem to be absent in L. sanfranciscensis. However, guanosine and adenosine as well as the corresponding nucleotides could be generated from IMP.

Although all genes necessary for the de novo synthesis of pyrimidines are present, L. sanfranciscensis is presumably auxotrophic for pyrimidines. The gene for dihydroorotase (EC 3.5.2.3; LSA_05890, LSA_05900), one of the five enzymes needed to generate UMP from carbamoyl-phosphate seems to be inactive due to a frameshift. Besides the dihydroorotase gene no additional pseudogenes are present in the pyrimidine metabolism of L. sanfranciscensis.

\section{Cofactors}

Similar to other lactobacilli, L. sanfranciscensis appears unable to synthesize most cofactors and vitamins like folate, thiamine, riboflavin, vitamine $\mathrm{B} 6$, nicotinate and nicotinamide. In silico analysis predicts that this organism can utilize both nicotinate and nicotinamide to generate NAD. However, this is only possible as two of the key enzymes, nicotinamidase (Ec 3.5.1.19) and nicotinate phosphoribosyltransferase (EC 2.4.2.11) are encoded by the plasmid pLS2 ( LSA_2p00220, LSA_2900230).

Although only one gene involved in cobalamine synthesis (cobyrinic acid a, c-diamide synthase, EC 6.3.5.11; LSA_2900630) was encoded by the sequenced strain L. sanfranciscensis TMW1.1304, growth experiments showed that 8 of 11 L. sanfranciscensis strains tested were able to grow on vitamin B12 free media (Difco ${ }^{\mathbb{R}}$ ) indicating that those strains were able to synthezise cobalamine de novo.

\section{Proteolytic system}

The predicted auxotrophy for 12 amino acids for L. sanfranciscensis was consistent with the presence of a large number of peptidases, proteases and transport systems for amino acids and peptides (Additional file 6). A complex proteolytic system ensures not only the supply with essential amino acids but also likely provides $L$. sanfranciscensis with a selective advantage in its protein-rich environment

Table 2 Comparison of abundance of cytoplasmatic permeases in relation to genome size and predicted auxotrophy for amino acids between different LAB

\begin{tabular}{|c|c|c|c|c|}
\hline Organism & Cytoplasmatic aminopeptidases & Genome size [Mbp] & Auxotrophic for amino acids & Reference \\
\hline L. plantarum WCFS1 & 19 & 3.31 & 3 & {$[24]$} \\
\hline L. sanfranciscensis TMW 1.1304 & 20 & 1.29 & 12 & This work \\
\hline L. acidophilus NCFM & 20 & 1.99 & 14 & {$[31]$} \\
\hline L. helveticus DPC 4571 & 24 & 2.08 & 16 & {$[60]$} \\
\hline L. johnsonii NC 533 & 25 & 1.99 & 20 & {$[61]$} \\
\hline L. casei ATCC 334 & 27 & 3.08 & 3 & {$[62]$} \\
\hline L. gasseri ATCC 33323 & 29 & 1.89 & 17 & {$[30]$} \\
\hline
\end{tabular}


as acquisition of amino acids from the environment is energetically more favourable than de novo synthesis [10].

The absence of an extracellular protease (prt) gene in the genome of L. sanfranciscensis reflects its high adaptation to and the associated dependency on sourdough. In contrast, dairy lactobacilli with comparable auxotrophy for amino acids like $L$. helveticus or L. acidophilus encode prt genes for proteinase production as milk only has low proteolytic activity and therefore degradation of casein to oligopeptides is a prerequisite for the growth of lactic acid bacteria in milk [31,32].

Peptides and amino acids present in the sourdough environment are internalized by peptide transporters and amino acid permeases. The L sanfranciscensis genome encodes a di-/tripeptide transporter $d t p T$ (LSA_04370) and a complete oligopeptide transport system Opp. All five genes of the Opp system $(o p p D, o p p F, o p p B, o p p C, o p p A)$ are organized in an operon (LSA_0280-LSA_0320). In addition to several amino acid transporters with unknown specificity, ABC transporters for glutamine $(g \ln H M P Q$, LSA_13380-13410), methionine (LSA_12550-12560, LSA_0940, LSA_08450) and cystine (tcyABC, LSA_01990, LSA_8550, LSA_8540, LSA_10490) are predicted. Additionally a lysine-specific permease (LSA_11550), a serinethreonine antiporter (steT, LSA_03230), an arginineornithine antiporter (arcD, LSA_12260), a choline-glycine betaine transporter (LSA_11780) and a $\gamma$-aminobutyrate permease (LSA_8760) are present.

L. sanfranciscensis has 20 genes encoding cytoplasmatic peptidases of different specificity to hydrolyze incorporated peptides into free amino acids. (Additional file 6). Many of the genes were described in L. sanfranciscensis previously [33], but the genome sequence included novel genes with homology to the pepB, pepD, pepE, pepM, $p e p O$, pep $Q$, and $p e p V$. Compared to other LAB no correlation between amino acid auxotrophy and content of cytoplasmatic peptidases can be observed.

\section{Regulators}

Based on the presence of conserved functional domains 2 two-component regulatory systems and 38 transcriptional regulators including five pseudogenes were predicted (Additional files 7 and 8). Compared to L. acidophilus NCFM that harbours 9 two-component regulatory systems, this is a quite low number of genes involved in gene regulation and might reflect adaptation to a stable and nutrient-rich environment, where less adaptive regulation is required [34][30]. Like for other sequenced lactobacilli the numerically predominant regulatory protein families are repressors, i. e. MarR (five members), AcrR (four members) and MerR (four members). Besides $\operatorname{csp} A$ and the 2 two-component regulatory systems only two transcriptional activators both belonging to the LysR family were predicted. L. sanfranciscensis has three genes encoding for sigma factors. Besides the primary sigma-factor rpoD (LSA_7720) two genes for rpoE (LSA_03460 and LSA_4550), a sigma factor involved in high temperature and oxidative stress response are present.

\section{Phage defense/restriction/modification systems}

CRISPR loci play a critical role in the adaptation and persistence of a microbial host in a particular ecosystem. The observed similarity between spacers and phage or plasmid sequences has led to the hypothesis that CRISPRs may provide resistance against foreign DNA determinants [35-39]. Using CRISPRFinder [36], a web tool to identify clustered regularly interspaced short palindromic repeats (CRISPR) we identified two CRISPR loci. A chromosomally located CRISPR/cas system consists of three cas genes followed by two 29 bp CRISPR spacers. Repeat length (36 bp) and sequence similarity indicates its belonging to the Lsal1 family. A plasmid-located CRISPR/cas system consists of 5 cas genes and a CRISPR with 14 spacers, where the 28 bp repeats are separated from the cas genes by an IS607-family transposase gene. Repeat size (29bp) and sequence as well as spacer size $(32 / 33 \mathrm{bp})$ are identical to L. brevis ATCC 367 CRISPR Ldbu1-family [40]. Only one other plasmid-encoded CRISPR/cas system was identified on the Enterococcus faecium pHT beta plasmid [41]. The repeat number in L. sanfranciscensis is below the average number of repeats per locus of 19.5 found in other LAB [40].

Very few phages of lactobacilli have been isolated from sourdough samples $[42,43]$ and only one phage active on $L$. sanfranciscensis was described [44]. BLAST analysis of spacer sequences resulted in the identification of only two significant hits for plasmid-encoded spacer 14 with $100 \%$ similarity to Lactococcus lactis plasmid pEW104 (AF097471) and for the chromosomal spacer 2 with $93 \%$ to L. plantarum plasmid pLTK2 (AB024514). No hit was found for a known phage sequence. While phage infections and spread in sourdough cultures may be hampered by the solid texture of the fermentative mass in batch systems the presence of CRISPR/cas may generally account for genetic stability of this strain in the sourdough environment making it a stable element over decades of fermentation.

\section{Mobile elements}

The presence of 111 transposases (including 25 pseudogenes) in IS elements in five different IS element families ( IS3, IS30, ISL3, IS200/605, IS256) represent $7.7 \%$ of the ORFs found and are, as a result of the small chromosome, found in higher proportion than in other lactobacilli. Thus, an idea of facilitated niche adaptation of a distinct Lactobacillus subpopulation by a relative increase of genome plasticity is supported. 


\section{Stress response}

Among genes related to heat, cold, acid, DNA damage and starvation genes related to the capability to respond to osmotic and oxidative stress are pronounced despite the small genome, suggesting that L. sanfranciscensis frequently faces such stresses. Generally, tolerance to oxygen of lactic acid bacteria requires the presence of catalase and/or NADH oxidases or several thiol-active enzyme systems including the thioredoxin-thioredoxin reductase couple, the glutathione-GshR system and a cyst(e)ine uptake and metabolism. On the basis of sequence similarity beside a NADH oxidase (LSA_05610) we identified in L. sanfranciscensis, a glutathione reductase (LSA_2p00270), a glutaredoxin-like protein (LSA_04700), two thioredoxin reductases (LSA_02530, LSA_05170), a putative thioredoxin peroxidase (LSA_09790), three thioredoxin-like proteins (LSA_08950, LSA_02610, LSA_06080) and a cyst(e) ine transport protein (LSA_08550). It was previously reported that a glutathione reductase negative mutant strain of L. sanfranciscensis DSM20451 ${ }^{\mathrm{T}}$ lost oxygen tolerance and exhibited a strongly decreased aerobic growth rate compared to either the growth rate under anaerobic conditions or that of the wild-type strain. Moreover aerobic growth was restored by the addition of cysteine [45]. In the majority of organisms glutathione is synthesized by the sequential action of $\gamma$-glutamylcysteine synthetase and glutathione synthetase, encoded by $g s h A$ and $g s h B$, respectively. The genome of L. sanfranciscensis contain no homolog of these two enzymes indicating that glutathione is probably to be imported from the medium. Actually, in the traditional backslopping sourdough process is a solid state fermentation with varying water activities and repeated mixing procedures frequently introduce oxygen. However, oxygen is readily used as electron acceptor in the reaction of $\mathrm{NADH}$ oxidase II, which directly produces water.

\section{Bacteriocins}

The production of inhibitory substances by sourdough LAB could provide another selective advantage for the producer strains [46]. Bacteriocins so far discovered from sourdough LAB and include the bacteriocins bavaricin A [47], plantaricin ST31 [48] and the bacteriocin-like inhibitory substance L. sanfranciscensis C57. [49].

No functionally active bacteriocin genes are found in the genome. Only two truncated genes sharing 100\% with papA encoding pediocin were found. Thus, bacteriocin production cannot be the reason for the long term competitiveness of this bacterium in sourdough.

\section{Plasmids and plasmid encoded traits}

Two plasmids pLS1 and pLS2 were present in strain TMW 1.1304. While plasmid-encoded traits for lactobacilli frequently include genes for sugar metabolism plasmids pLS1 and pLS2 harbour genes involved in nucleotide/NADH metabolism and are further characterized by the presence of many orfs encoding transposases.

The total DNA sequence of pLS1 consists of 58,739 bp with a GC content of $37.6 \%$ encoding 59 orfs. Two genes encoding Rep B (replication associated replication protein) and RepA are homologous to the corresponding genes of the L. brevis plasmid pLB925A04 that is a theta type replication plasmid of the pAM $\beta$-1-family [50]. A truncated dextransucrase (LSA_2p00510) is discussed above. PLS1 contains a CRISPR/cas locus further supporting the importance of this function even at an enhanced copy number (see above).

The total DNA sequence of pLS2 consists of 18,715 bp with a GC content of $36.1 \%$ and encodes 19 orfs. The replication protein RepB shows $80 \%$ similarity to a RepB protein of Enterococcus faecium E1636 (EMBL EFF23229.1) and $40 \%$ to a RepB of plasmid pLTK13 (EMBL BAG67041), a rolling circle replicating plasmid of L. plantarum L137. Replication of the lagging strand of $\mathrm{RC}$ plasmids initiates from their single-strand origins (SSOs). SSOs have a high potential for intrastrand pairing and based on their secondary structures, several types of SSOs have been identified [51,52]. PLS2 contains a palindromic region (position 21-58) whose secondary structure is similar to the ssoA-type origin. A restriction / modification system consists of a cytosinespecific methyltransferase followed by a restriction endonuclease gene similar to the McrBC restriction endonuclease system of Rhodobacter capsulatus ATCC BAA-309 (EMBL ADE85042 .1).

\section{Material and methods}

\section{Strain selection, strain purification and DNA isolation}

For sequencing a strain without any laboratory transfers was selected to ensure sequencing of a truly sourdough adapted clone. Therefore, the strain was isolated on mMRS [53] at $30^{\circ} \mathrm{C}$ from "Böcker Reinzuchtsauer", a rye sourdough starter, which is now propagated for 100 years in the same tradition, and only propagated on laboratory media to obtain enough DNA for sequencing. Dilutions of a sourdough sample were spread directly on mMRS agar plates. Plates were incubated under anaerobic conditions at $30{ }^{\circ} \mathrm{C}$ for 3-5 days. Genomic DNA was isolated with the EZNA DNA reagent set (Omega Bio-Tek) according the provided protocol for Gram positive bacteria.

\section{Sequencing strategy}

Sequencing was done in a combined Sanger/454-pyrosequencing approach. 454 sequencing resulted in 187,929 reads with an average read length of 250 nucleotides giving $~ 45 \mathrm{Mbp}$ sequencing data corresponding to a 33-fold coverage. In addition 10,000 genomic fragments with typically $3 \mathrm{~kb}$ to $5 \mathrm{~kb}$ inserts were cloned into the TOPO 
TA vector (Quiagen, Hilden) and sequenced on an ABI 3730 capillar sequencer from both ends. The ABI sequences resulted in 19.569 reads corresponding to an additional coverage of 13 fold. Remaining gaps were closed by sequences generated on gap-spanning PCR products by an ABI 3730 capillary sequencer. The overall quality was set to a minimum confidence of PHRED 45 for the complete genome.

This genome project has been deposited in the European Molecular Biology Laboratory (EMBL)/Gen- Bank under the accession numbers CP002461 (chromosome), CP002462 (pLS1) and CP002463 (pLS2). The version described in this paper is the first version. Prediction of protein encoding sequences and open reading frames (ORFs) were initially accomplished with PEDANT software suite [54]. The PEDANT genome database provides exhaustive annotation of nearly 3000 publicly available eukaryotic, eubacterial, archaeal and viral genomes. Gene prediction was performed with GenMark 2.8 [55] and Glimmer 3.0 [56] as implemented in the Pedant software suite. All orf predictions were verified and modified by a blasting orfs to NCBI nrdb. Additionally, the predicted start codons of all ORFs were inspected manually using the Artemis program [57]. Clustered regularly interspaced short palindromic repeats (CRISPR) were identified with the web tool CRISPRFinder [36].

\section{Phylogenetic tree}

A phylogenetic tree on the basis of a multiple $16 \mathrm{~S}$ rDNA alignment based similarity matrix was constructed by the neighbour-joining method [58] using the software package Bionumerics v6.5 (Applied Maths, Belgium). Unknown bases were discarded for the analyses. Bootstrapping analysis was undertaken to test the statistical reliability of the topology of the neighbour-joining tree using 100 bootstrap resamplings of the data

\section{Exopolysaccharide analysis}

For production of EPS strain TMW 1.1304 was grown on mMRS containing $80 \mathrm{~g} \mathrm{l}-1$ of sucrose at $30^{\circ} \mathrm{C}$ for 24 h. To analyze the type of the polymer the EPS was precipitated by adding two volumes of ethanol (99\%) and incubation at $4{ }^{\circ} \mathrm{C}$ for $18 \mathrm{~h}$. EPS was hydrolyzed with perchloric acid at $100^{\circ} \mathrm{C}$ for $3 \mathrm{~h}$ and sugar monomers were analyzed with HPLC as described by Waldherr et al. 2008 [28].

\section{Additional material}

Additional file 1: Protein length distribution and average orf length of L. sanfranciscensis TMW 1.1304 compared to other lactobacilli genomes. Data were extracted from the PEDANT 3 database (Walter et al. 2009)
Additional file 2: List of genomes used for the analysis of the rRNA operon density.

Additional file 3: List of genomes with rRNA gene densities above 2.9 per Mbp.

Additional file 4: List of Lactobacillus sanfranciscensis genes which are translationally optimized with respect to codon usage bias.

Additional file 5: In silico analysis of the genome of $\mathrm{L}$. sanfranciscensis TMW 1.1304 for ORFs putatively involved in the utilization of different carbon sources

Additional file 6: Summary of the L. sanfranciscensis cytoplasmatic proteases and peptidases, and their cleavage specificities, gene names and relative abundance in the genome. The ' $\mid$ ' indicates the cleavage site.

Additional file 7: Transcriptional regulators witin the genome of Lactobacillus sanfranciscensis TMW 1.1304

Additional file 8: Presence of genes for transcriptional regulators and two-component regulatory systems in different lactobacilli genomes

\section{Acknowledgements}

We thank T. Rattei and P. Tischler, Lehrstuhl für Bioinformatik, Technische Universität München, Germany, for their technical support in bioinformatics analyses. Assistance in rRNA sequence analysis by W. Ludwig, Technische Universität München, and genome-wide codon usage analysis by R. Merkl, Universität Regensburg, Germany, is gratefully acknowledged.

This article has been published as part of Microbial Cell Factories Volume 10 Supplement 1, 2011: Proceedings of the 10th Symposium on Lactic Acid Bacterium. The full contents of the supplement are available online at http:// www.microbialcellfactories.com/supplements/10/S1.

\section{Author details}

${ }^{1}$ Lehrstuhl für Technische Mikrobiologie, Germany. ${ }^{2}$ Lehrstuhl für Mikrobiologie, Technische Universität München, 85350 Freising, Germany. ${ }^{3}$ Laboratorium für Genomanalyse, Institut für Mikrobiologie und Genetik, Georg-August-Universität Göttingen, Germany. ${ }^{4}$ Ernst Böcker GmbH \& Co KG, Minden, Germany.

\section{Competing interests}

The authors declare that they have no competing interests.

Published: 30 August 2011

\section{References}

1. Vogel RF, Gänzle MG: Bread is an essential part of human nutrition and culture. Food Microbiology 2009, 26:665-671.

2. Kline L, Sugihara TF: Microorganisms of the San Francisco sour dough bread process. II. Isolation and characterization of undescribed bacterial species responsible for the souring activity. App/ Microbiol 1971, 21:459-465.

3. Weiss N, Schillinger U: Lactobacillus sanfrancisco sp. nov., nom. rev. System Appl Microbiol 1984, 5:230-232.

4. Trüper $\mathrm{H}-\mathrm{G}, \mathrm{L}$ DC: Taxonomic note: necessary correction of specific epithets formed as substantives (nouns) "in apposition. Int I Syst Bacteriol 1997, 47:908-909.

5. Gobbetti M, Corsetti A: Lactobacillus sanfrancisco a key sourdough lactic acid bacterium: a review. Food Microbiology 1997, 14:175-187.

6. Vogel RF, Ehrmann MA, Ganzle MG: Development and potential of starter lactobacilli resulting from exploration of the sourdough ecosystem. Antonie Van Leeuwenhoek 2002, 81:631-638.

7. Groeneveld WH, Van Reenen CA, Todorov SD, DuToit M, Witthuhn RC, Holzapfel WH, Dicks LMT: Identification of lactic acid bacteria from vinegar fruit flies based on their phenotypic and genotypic characteristics. Am J Enol Vitic 2006, 57:519-525.

8. Thiele C, Gaenzle MG, Vogel RF: Contribution of sourdough lactobacilli, yeast, and cereal enzymes to the generation of amino acids in dough relevant for bread flavour. Cereal Chemistry 2002, 79:45-51. 
9. Gallo G, De Angelis M, MCSweeney PLH, Corbo MR, Gobbetti M: Partial purification and characterization of an X-prolyl dipeptidyl aminopeptidase from Lactobacillus sanfranciscensis CB1. Food Chemistry 2005, 91:535-544.

10. Gobbetti M, Smacchi E, Corsetti A: The proteolytic system of Lactobacillus sanfrancisco CB1: purification and characterization of a proteinase, a dipeptidase, and an aminopeptidase. Appl Environ Microbiol 1996, 62:3220-3226.

11. De Angelis LM, Rossi J, Servili M, Fox PF, Rollán G, Gobbetti M: Arginine catabolism by sourdough lactic acid bacteria: purification and characterization of the arginine deiminase pathway enzymes from Lactobacillus sanfranciscensis CB. Applied and Environmental Microbiology 2002, 68:6193-6201.

12. Tieking M, Ehrmann MA, Vogel RF, Ganzle MG: Molecular and functional characterization of a levansucrase from the sourdough isolate Lactobacillus sanfranciscensis TMW 1.392. Appl Microbiol Biotechnol 2005, 66:655-663.

13. Zapparoli G, Toriani S, Dellaglio F: Differentiation of Lactobacillus sanfrancicensis strains by randomly amplified polymorphic DNA and pulsed-field gel electrophoresis. FEMS Microbiol Lett 1998, 166:325-332.

14. Macklaim JM, Gloor GB, Anukam KC, Cribby S, Reid G: At the crossroads of vaginal health and disease, the genome sequence of Lactobacillus iners AB-1. Proceedings of the National Academy of Sciences 2010 [http://www. pnas.org/cgi/doi/10.1073/pnas.1000086107].

15. Tamames J, Gil R, Latorre A, Pereto J, Silva FJ, Moya A: The frontier between cell and organelle: genome analysis of Candidatus Carsonella ruddii. BMC Evol Biol 2007, 7:181.

16. Condon C, Liveris D, Squires C, Schwartz I, Squires CL: rRNA operon multiplicity in Escherichia coli and the physiological implications of rrn inactivation. J Bacteriol 1995, 177:4152-4156.

17. Klappenbach JA, Dunbar JM, Schmidt TM: rRNA operon copy number reflects ecological strategies of bacteria. Appl Environ Microbiol 2000, 66:1328-1333.

18. Andersson SGaK, C G: Codon preferences in free-living microorganisms. Microbiol Rev 1990, 54:198-210.

19. Sharp PM, Bailes E, Grocock RJ, Peden JF, Sockett RE: Variation in the strength of selected codon usage bias among bacteria. Nucleic Acids Res 2005, 33:1141-1153.

20. Van Mandach C, Merkl R: Genes optimized by evolution for accurate and fast translation encode in Archaea and Bacteria a broad and characteristic spectrum of protein functions. BMC Genomics 2010, 11:617.

21. Stolz P, Böcker G, Hammes WP, Vogel RF: Utilization of electron acceptors by lactobacilli isolated from sourdough. I. Lactobacillus sanfrancisco. $Z$ Lebensm Unters Forsch 1995, 201:91-96.

22. Zhang C, Ganzle MG: Metabolic pathway of a-ketoglutarate in Lactobacillus sanfranciscensis and Lactobacillus reuteri during sourdough fermentation. Journal of Applied Microbiology 2010, 109:1301-1310.

23. Radler $F$, Brohl $K$ : The metabolism of several carboxylic acids by lactic acid bacteria. Z Lebensm Unters Forsch 1984, 179:228-231

24. Kleerebezem M, Boekhorst J, van Kranenburg R, Molenaar D, Kuipers OP Leer R, Tarchini R, Peters SA, Sandbrink HM, Fiers MW: Complete genome sequence of Lactobacillus plantarum WCFS1. Proc Natl Acad Sci U S A 2003, 100:1990-1995

25. Rico J, Yebra MJ, Perez-Martinez G, Deutscher J, Monedero V: Analysis of Idh genes in Lactobacillus casei BL23: role on lactic acid production. $J$ Ind Microbiol Biotechnol 2008, 35:579-586.

26. Waldherr F, Vogel RF: Commercial Exploitation of Homoexopolysaccharides in Non-dairy Food Systems. In Bacterial Exopolysaccharides - current innovations and future trends. Caister Academic Press; Ulrich M 2009:

27. Tieking M, Kuhnl W, Ganzle MG: Evidence for formation of heterooligosaccharides by Lactobacillus sanfranciscensis during growth in wheat sourdough. J Agric Food Chem 2005, 53:2456-2461.

28. Waldherr FW, Meissner D, Vogel RF: Genetic and functional characterization of Lactobacillus panis levansucrase. Arch Microbio/ 2008 190:497-505.

29. Korakli M, Vogel RF: Structure/function relationship of homopolysaccharide producing glycansucrases and therapeutic potential of their synthesised glycans. Appl Microbiol Biotechnol 2006, 71:790-803.
30. Azcarate-Peril MA, Altermann E, Goh YJ, Tallon R, Sanozky-Dawes RB, Pfeiler EA, O'Flaherty S, Buck BL, Dobson A, Duong T: Analysis of the genome sequence of Lactobacillus gasseri ATCC 33323 reveals the molecular basis of an autochthonous intestinal organism. Appl Environ Microbiol 2008, 74:4610-4625.

31. Altermann E, Russell WM, Azcarate-Peril MA, Barrangou R, Buck BL, McAuliffe O, Souther N, Dobson A, Duong T, Callanan M: Complete genome sequence of the probiotic lactic acid bacterium Lactobacillus acidophilus NCFM. Proc Natl Acad Sci U S A 2005, 102:3906-3912.

32. Pastar I, Begovic J, Lozo J, Topisirovic L, Golic N: Casitone-dependent transcriptional regulation of the prtP and prtM genes in the natural isolate Lactobacillus paracasei subsp. paracasei. Folia Microbiol (Praha) 2007, 52:577-584.

33. Vermeulen N, Pavlovic M, Ehrmann MA, Ganzle MG, Vogel RF: Functional characterization of the proteolytic system of Lactobacillus sanfranciscensis DSM 20451T during growth in sourdough. Appl Environ Microbiol 2005, 71:6260-6266.

34. Stock AM, Robinson VL, Goudreau PN: Two-component signal transduction. Annu Rev Biochem 2000, 69:183-215.

35. Bolotin A, Quinquis B, Sorokin A, Ehrlich SD: Clustered regularly interspaced short palindrome repeats (CRISPRs) have spacers of extrachromosomal origin. Microbiology 2005, 151:2551-2561.

36. Grissa I, Vergnaud G, Pourcel C: CRISPRFinder: a web tool to identify clustered regularly interspaced short palindromic repeats. Nucleic Acids Res 2007, 35:52-57.

37. Horvath P, Romero DA, Coute-Monvoisin AC, Richards M, Deveau H, Moineau S, Boyaval P, Fremaux C, Barrangou R: Diversity, activity, and evolution of CRISPR loci in Streptococcus thermophilus. J Bacteriol 2008, 190:1401-1412.

38. Makarova KS, Grishin NV, Shabalina SA, Wolf YI, Koonin EV: A putative RNAinterference-based immune system in prokaryotes: computational analysis of the predicted enzymatic machinery, functional analogies with eukaryotic RNAi, and hypothetical mechanisms of action. Biol Direct 2006, 1:7.

39. Mojica FJ, Diez-Villasenor C, Garcia-Martinez J, Soria E: Intervening sequences of regularly spaced prokaryotic repeats derive from foreign genetic elements. J Mol Evol 2005, 60:174-182.

40. Horvath P, Coute-Monvoisin AC, Romero DA, Boyaval P, Fremaux C, Barrangou R: Comparative analysis of CRISPR loci in lactic acid bacteria genomes. Int J Food Microbiol 2009, 131:62-70.

41. Tomita H, Ike Y: Genetic analysis of transfer-related regions of the vancomycin resistance Enterococcus conjugative plasmid pHTbeta: identification of oriT and a putative relaxase gene. J Bacteriol 2005, 187:7727-7737.

42. Foschino R, Perrone F, Galli A: Characterization of two virulent Lactobacillus fermentum bacteriophages isolated from sour dough. J Appl Bact 1995, 79:677-683.

43. Foschino R, Galli A, Pagani A, Ottogalli G: Isolation and characterization of bacteriophages active on heterofermentative lactobacilli in sourdoughs. Microbiol Alim Nutr 1996, 14:15-22.

44. Foschino R, Venturelli E, Picozzi C: Isolation and characterization of a virulent Lactobacillus sanfranciscensis bacteriophage and its impact on microbial population in sourdough. Curr Microbiol 2005, 51:413-418.

45. Jaensch A, Korakli M, Vogel RF, Ganzle MG: Glutathione reductase from Lactobacillus sanfranciscensis DSM20451 ${ }^{\top}$ : contribution to oxygen tolerance and thiol exchange reactions in wheat sourdoughs. Appllied and Environmental Microbiology 2007, 73:4469-4476.

46. Messens W, DeVuyst L: Inhibitory substances produced by Lactobacilli isolated from sourdoughs-a review. Int J Food Microbiol 2002, 72:31-43

47. Larsen AG, Vogensen FK, Josephsen J: Antimicrobial activity of lactic acid bacteria isolated from sour doughs: purification and characterization of bavaricin A, a bacteriocin produced by Lactobacillus bavaricus MI401. J Appl Bacteriol 1993, 75:113-122.

48. Todorov S, Onno B, Sorokine O, Chobert JM, Ivanova I, Dousset X: Detection and characterization of a novel antibacterial substance produced by Lactobacillus plantarum ST 31 isolated from sourdough. Int J Food Microbiol 1999, 48:167-177.

49. Corsetti A, Gobbetti M, Smacchi E: Antibacterial activity of sourdough lactic acid bacteria: isolation of a bacteriocin-like inhibitory substance from Lactobacillus sanfrancisco. Food microbiology 1996, 13:447-456. 
50. Wada T, Noda M, Kashiwabara F, Jeon HJ, Shirakawa A, Yabu H, Matoba Y, Kumagai T, Sugiyama M: Characterization of four plasmids harboured in a Lactobacillus brevis strain encoding a novel bacteriocin, brevicin 925A, and construction of a shuttle vector for lactic acid bacteria and Escherichia coli. Microbiology 2009, 155:1726-1737.

51. Khan SA: Rolling-circle replication of bacterial plasmids. Microbiol Mol Biol Rev 1997, 61:442-455

52. Khan SA: Plasmid rolling-circle replication: recent developments. Mol Microbiol 2000, 37:477-484

53. Stolz P, Hammes WP, Vogel RF: Maltose-phosphorylase and hexokinase activity in lactobacilli from traditionally prepared sourdoughs. Adv Food Sci 1996, 18:1-6.

54. Walter MC, Rattei T, Arnold R, Guldener U, Munsterkotter M, Nenova K, Kastenmuller G, Tischler P, Wolling A, Volz A: PEDANT covers all complete RefSeq genomes. Nucleic Acids Res 2009, 37:D408-411.

55. Borodovsky M, Mills R, Besemer J, Lomsadze A: Prokaryotic gene prediction using GeneMark and GeneMark.hmm. Curr Protoc Bioinformatics 2003, 5, Chapter 4:Unit4.

56. Delcher AL, Bratke KA, Powers EC, Salzberg SL: Identifying bacterial genes and endosymbiont DNA with Glimmer. Bioinformatics 2007, 23:673-679.

57. Rutherford K, Parkhill J, Crook J, Horsnell T, Rice P, Rajandream MA, Barrell B: Artemis: sequence visualization and annotation. Bioinformatics 2000 16:944-945.

58. Saitou N, Nei M: The neighbor-joining method: a new method for reconstructing phylogenetic trees. Molecular Biology Evolution 1987, 4:406-425

59. Morita H, Toh H, Fukuda S, Horikawa H, Oshima K, Suzuki T, Murakami M, Hisamatsu S, Kato Y, Takizawa T: Comparative genome analysis of Lactobacillus reuteri and Lactobacillus fermentum reveal a genomic island for reuterin and cobalamin production. DNA Res 2008, 15:151-161.

60. Callanan M, Kaleta P, O'Callaghan J, O'Sullivan O, Jordan K, McAuliffe O, Sangrador-Vegas A, Slattery L, Fitzgerald GF, Beresford T, Ross RP: Genome sequence of Lactobacillus helveticus, an organism distinguished by selective gene loss and insertion sequence element expansion. $J$ Bacteriol 2008, 190:727-735

61. Pridmore RD, Berger B, Desiere F, Vilanova D, Barretto C, Pittet AC, Zwahlen MC, Rouvet M, Altermann E, Barrangou R, et al: The genome sequence of the probiotic intestinal bacterium Lactobacillus johnsonii NCC 533. Proc Natl Acad Sci U S A 2004, 101:2512-2517.

62. Cai H, Thompson R, Budinich MF, Broadbent JR, Steele JL: Genome sequence and comparative genome analysis of Lactobacillus casei: insights into their niche-associated evolution. Genome Biol Evol 2009, 1:239-257.

doi:10.1186/1475-2859-10-S1-S6

Cite this article as: Vogel et al:: Genomic analysis reveals Lactobacillus sanfranciscensis as stable element in traditional sourdoughs. Microbial Cell Factories 2011 10(Suppl 1):S6.

\section{Submit your next manuscript to BioMed Central and take full advantage of:}

- Convenient online submission

- Thorough peer review

- No space constraints or color figure charges

- Immediate publication on acceptance

- Inclusion in PubMed, CAS, Scopus and Google Scholar

- Research which is freely available for redistribution 